\title{
Navigation Expertise and the Human Hippocampus: A Structural Brain Imaging Analysis
}

\author{
Eleanor A. Maguire, ${ }^{1 *}$ Hugo J. Spiers, ${ }^{2}$ \\ Catriona D. Good, ${ }^{1}$ Tom Hartley, ${ }^{2}$ \\ Richard S.J. Frackowiak, ${ }^{1}$ and Neil Burgess ${ }^{2}$ \\ ${ }^{1}$ Wellcome Department of Imaging Neuroscience, \\ Institute of Neurology, University College London, \\ London, United Kingdom \\ ${ }^{2}$ Institute of Cognitive Neuroscience and Department of \\ Anatomy and Developmental Biology, University College \\ London, London, United Kingdom
}

\begin{abstract}
Grey matter volume in the posterior hippocampus of London taxi drivers is greater than in age-matched controls, and the size of this increase correlates positively with time spent taxi driving (E.A. Maguire et al., 2000. Proc Natl Acad Sci U S A 97: 4398-4403). This change suggests that increased posterior hippocampal grey matter volume is acquired in response to increased taxi driving experience, perhaps reflecting their detailed representation of the city. However, an alternate hypothesis is that the difference in hippocampal volume is instead associated with innate navigational expertise, leading to an increased likelihood of becoming a taxi driver. To investigate this possibility, we used structural brain imaging and voxel-based morphometry (VBM) to examine a group of subjects who were not taxi drivers. Despite this group showing a wide range of navigational expertise, there was no association between expertise and posterior hippocampal grey matter volume (or, indeed, grey matter volume throughout the brain). This failure to find an association between hippocampal volume and navigational expertise thus suggests that structural differences in the human hippocampus reflect the detail and/or duration of use of the spatial representation acquired, and not innate navigational expertise per se. Hippocampus 2003;13:208-217. ๑ 2003 Wiley-Liss, Inc.
\end{abstract}

KEY WORDS: hippocampal grey matter; VBM; virtual reality; wayfinding

\section{INTRODUCTION}

The ability to navigate through the environment is essential for everyday living. Predicting a person's ease at navigation is difficult, however, as many factors contribute to navigation expertise. Some of these might be termed

Grant sponsor: Wellcome Trust; Grant sponsor: Medical Research Council, UK.

*Correspondence to: Eleanor Maguire, Wellcome Department of Imaging Neuroscience, Institute of Neurology, 12 Queen Square, London WC1N 3BG, UK. E-mail: e.maguire@fil.ion.ucl.ac.uk

Accepted for publication 3 June 2002 Published online

DOI 10.1002/hipo.10087 "external" (Prestopnik and Roskos-Ewoldsen, 2000), such as the availability and density of meaningful landmarks, or the pattern of streets and intersections (O’Neill, 1992; Tlauka and Wilson, 1994). Other "internal" factors include the level of familiarity with the environment, the cognitive strategies used while navigating, and the sex and age of the navigator (Siegal and White, 1975; O'Keefe and Nadel, 1978; Hermer and Spelke, 1994; Sandstrom et al., 1998). Given its multifaceted nature, there is little agreement on the best way to measure navigation expertise formally (for examples of tasks used, see Prestopnik and Roskos-Ewoldsen, 2000). One obvious and direct approach is to get subjects to find their way in an environment or to describe routes they would take. For real environments, it is difficult across subjects to control for factors such as familiarity and range of exploration. However, one group where such factors are more homogeneous is among taxi drivers.

Taxi drivers in London (England) engage in extensive training to learn how to navigate between thousands of places in the city. This training is colloquially known as "The Knowledge" and takes two years on average. In order to be licensed to operate, it is necessary to pass a very stringent set of police examinations. A previous functional neuroimaging study documented activation of the right posterior hippocampus in London taxi drivers associated with their successful recall of complex routes around the city (Maguire et al., 1997). Controlling for familiarity and range of exploration in non-taxi drivers, the use of virtual reality environments has more recently become a significant feature of navigation research (Maguire et al., 1999; Burgess and King, 2001). Further evidence for the engagement of the right hippocampus by successful navigation has been found in non-taxi driver subjects who navigated to destinations within a virtual reality town during functional neuroimaging (Maguire et al., 1998). Successful trials (i.e., where 
a goal destination was reached) compared with "lost" trials (where subjects failed to find their way successfully) resulted in greater activation of the right posterior hippocampus. Interestingly, the better the angular accuracy of the paths to the goal places, the more active the right hippocampus, but in this instance with a more anterior activation peak. No correlations with anterior hippocampal activity were found with route recall in the taxi drivers; this is perhaps unsurprising as all of the taxi drivers performed with a very high level of accuracy (Maguire et al., 1997). To our knowledge, no other functional neuroimaging study of navigation has examined path accuracy, while in line with Maguire et al. $(1997,1998)$ several other studies have reported right posterior hippocampal activation associated with successful navigation (e.g., Grön et al., 2000; Ghaem et al., 1997). Overall, functional neuroimaging studies of navigation suggest that the right posterior hippocampus is involved in some way in successful navigation, while activity in the right anterior hippocampus seems to be more specifically indexing path accuracy.

In the light of increased hippocampal volume reported in small mammals and birds who engage in behaviour requiring spatial memory (Lee et al., 1998), the taxi drivers were examined further for structural brain changes associated with their extensive navigation experience (Maguire et al., 2000). Using VBM (Ashburner and Friston, 2000; Good et al., 2001a,b), an automated procedure for whole brain analysis of structural magnetic resonance imaging (MRI) scans, the posterior hippocampi of taxi drivers were found to have significantly greater grey matter volume relative to non-taxi driver subjects. A more anterior hippocampal region had greater grey matter volume in control subjects compared with taxi drivers. Right hippocampal grey matter volume correlated with the amount of time spent as a taxi driver (positively in the posterior and negatively in the anterior hippocampus). This right posterior hippocampal VBM finding corresponds closely with the functional neuroimaging data in taxi drivers (Maguire et al., 1997) and the other studies (Grön et al., 2000; Ghaem et al., 1997) where the right posterior hippocampus was active during successful navigation. The above findings led Maguire et al. (2000) to suggest that there may be a capacity for local plastic change in the structure of the healthy adult human brain in response to environmental demands, and that in particular the right posterior hippocampus may be the storehouse of the spatial representation of an environment, able to expand to accommodate its elaboration.

Based on the taxi driver findings, the proposal is that the hippocampal volume changes were acquired, and reflect the nature of the taxi drivers' detailed representation of the city. However, an alternate hypothesis is that the differences in hippocampal volume are instead associated with innate navigational expertise. Taxi drivers would thus have increased hippocampal grey matter volume because innately expert navigators may be more likely to become taxi drivers (Maguire et al., 2000; Terrazas and McNaughton, 2000). To investigate such a hypothesis requires the examination of grey matter in non-taxi driver navigation experts. If increased hippocampal grey matter volume is found to be taxi driver-specific, this would be further evidence for the association between hippocampal structure and the nature of the representation of a largescale space (Maguire et al., 2000). It would further suggest that functional neuroimaging studies of navigation success are not confounded by structural brain differences.

In the present study we used VBM to examine the structural MRI scans of non-taxi drivers and whether innate navigation expertise was associated with grey matter volume differences anywhere in the brain, particularly the hippocampus. In order to control for the factors of sex and familiarity, the subjects were all right-handed males and an unfamiliar virtual reality town was the setting for the navigation. The town was similar to that used in the previous functional neuroimaging study of Maguire et al. (1998), offering a controlled means to test navigation expertise whilst preserving many of the real-world qualities of actual navigation. Tests based in and on this virtual town are apparently sensitive to hippocampal function and structural integrity. Right posterior hippocampal activity was found to be associated with successful navigation in this town during functional neuroimaging (Maguire et al., 1998). Performance on tests based in and on this VR town has been shown to be compromised in patients who had undergone right temporal lobectomy, but relatively intact after left temporal lobectomy (Spiers et al., 2001a). Also, a patient with reportedly selective bilateral hippocampal damage (Jon, Vargha-Khadem et al., 1997; Gadian et al., 2000) was also impaired on the town-based tasks (Spiers et al., 2001b). Given that path accuracy may be more sensitive to the anterior hippocampus, we also included the other tests based on the virtual town performed by the patients reported in Spiers et al. $(2001 \mathrm{a}, \mathrm{b})$. Furthermore, it was planned to examine the correlations between the various tests to judge whether they were tapping different aspects of navigation performance.

\section{MATERIALS AND METHODS}

A total of 26 right-handed (non-taxi driver) males participated (Table 1). All subjects gave informed written consent in accordance with the local medical ethics committee. Mean age of subjects was 25.77 years, range $18-56$, and all subjects had healthy medical, neurological and psychiatric profiles. The full-scale IQs of participants were average or above average, estimated using the National Adult Reading Test (NART) (Nelson and Wilson, 1991) (Table 1). All except two subjects had prior experience of playing video games. One subject felt uncomfortable (nausea) viewing the virtual town, an occasional side effect of the optic-flow (Regan and Price, 1994). This patient did not explore further or perform any of the tasks related to the town. Owing to time constraints on two other subjects' availability, they were not tested on the verbal memory test, and one was not tested on the standardised topographical memory test.

\section{Behavioural Tasks}

Subjects performed the tasks either before or after the MRI scan (order of scan and tasks was random). A virtual reality town provided the environment about which the subjects were tested. The town was designed and constructed by Neil Burgess, using the commercially available video game Duke Nukem 3D ( $($ 3D 
TABLE 1.

Summary Scores from the Behavioural Measures

\begin{tabular}{|c|c|c|c|c|}
\hline Task & Measure & Mean & SD & Range \\
\hline NART $(n=26)$ & FSIQ equivalent & 112 & 6.84 & 95-124 \\
\hline \multicolumn{5}{|l|}{ Virtual town $(\mathrm{n}=25)$} \\
\hline Average angular accuracy & Degrees & 134.65 & 7.32 & $120-146.3$ \\
\hline \multicolumn{5}{|c|}{ Standardised Topographical Memory Test $(\mathrm{n}=25)$} \\
\hline CTRMT & Number correct out of 30 & 26.92 & 3.03 & $19-30$ \\
\hline
\end{tabular}

Standardised Verbal Memory Test $(\mathrm{n}=24)$

AMIPB story recall

Score out of a possible 56

Immediate

39.33

10.89

$17-54$

Delayed

36.63

9.71

$17-52$

SD, standard deviation; NART, National Adult Reading Test; CTRMT, Camden Topographical Recognition Memory Test; AMIPB, Adult Memory and Information Processing Battery.

${ }^{a}$ Higher score is poorer.

Realms Entertainment, Apogee Software Ltd. Garland TX) with the editor provided (Build $\odot$ 3D Realms Entertainment). It was adapted from the virtual town used in the study of Maguire et al. (1998); see its use also in Spiers et al. (2001a,b) and Burgess et al. (2001), and example views of the town in any of the aforementioned papers. Briefly, the town consisted of a main street intersected by a crossroad with various interior locations, including, for example, a cinema, a bookshop, a bar and an underground train station. The town was sufficiently complex to provide a large number of possible routes from one location to another. The town was displayed to the subjects at a frame rate of $21 \mathrm{~Hz}$ on a desktop PC with a 19-inch screen. Subjects used the cursor keys of a keyboard to maneuver within the town. They were instructed to explore until they felt they were ready to be tested on their memory for the town (and had been observed by the experimenter to visit all locations). Exploration was self-paced; subjects were required to explore for a minimum of $15 \mathrm{~min}$, but not more than $60 \mathrm{~min}$. Subjects were not warned that they would be tested on their ability to draw a map of the town, but were informed that they would be tested on their navigation and memory for the town. Subjects were tested on the following tasks in the order described.

\section{Navigation}

Subjects had to navigate between 10 different locations in the town using the most direct route available. They were shown a picture of a target location that was continuously present while they were navigating to that location. When subjects reached the location, they were presented a new picture and asked to repeat the process until they had navigated to each of the 10 places. These target locations were the same for each subject, evenly distributed across the town, never visible from the starting view, and varied in their relative proximity and difficulty to find. As detailed in Maguire et al. (1998), the subject's heading direction $\theta_{\mathrm{h}}$ (in degrees) and the direction of the current destination $\theta_{\mathrm{d}}$ was calculated at each virtual meter along the subject's trajectory. The absolute difference $\left|\theta_{\mathrm{h}}-\theta_{\mathrm{d}}\right|$ was found at each meter and was averaged over the task to give $A=180-<\left|\theta_{\mathrm{h}}-\theta_{\mathrm{d}}\right|>$ as a measure of the average angular accuracy of the heading. Theoretically, accuracy scores may vary from 0 (always moving directly away from the current destination) through 90 degrees (moving randomly) to 180 degrees (always moving directly towards the current destination). In practice, an accuracy score of 160 degrees is hard to exceed because of content of the environment (e.g., buildings).

\section{Environmental scene recognition}

Forced choice between pairs of scenes was used to test memory for the locations visited by the subject during exploration and navigation (see also Spiers et al., 2001a). Twenty pairs were tested. One of the scenes in each pair (the target) was a view of a location from the virtual town. Note that the subject was unlikely to have experienced the exact scene used and some of these were taken from viewpoints that subjects had never been able to reach. Thus, this scene recognition task differs from more standard tests, in that it requires the identification of scenes that have not been explicitly studied. Instead, successful discrimination of target views probably requires retrieval of abstracted environmental information, such as layout, that is dependent on the hippocampus (support for this comes from Spiers et al., 2001a, where the hippocampal damaged patient was impaired on this task). The other scenes (the foils) were created by altering the town or by creating new virtual locations. 
Some of these foil views had novel objects, novel textures and novel geometry. Other foils had familiar objects, familiar surface textures and similar geometry but were spatially rearranged so as to be inconsistent with the original town. Responding was self-paced.

\section{Map drawing}

Map drawing was assessed in a quantitative manner with a computer program (designed by T. Hartley; for full description, see Spiers et al., 2001a). A $10 \times 10$ grid and a set of 10 icons representing locations in the town were displayed on the computer monitor. Subjects were required to move icons onto the grid, to form a map of the town. After all 10 icons had been placed, subjects could opt to stop or to continue arranging the icons until they were satisfied. An ideal map was constructed that reflected the true layout of the town. Each subject's completed map was scored by calculating the error in the distance between all pairs of icons as a fraction of the mean distance between the icons in the ideal and subject's map. Thus, the higher the error score, the more erroneous the map. This measure is independent of the map's orientation. Scaling each subject's map to match the ideal map achieved independence from map size.

\section{Standardised topographical memory test}

A standardised and clinically used test of scene recognition was also administered to subjects for comparison with the experimental virtual town tasks. The Camden Topographical Recognition Memory Test (CTRMT) (Warrington, 1996) involves presentation of 30 colour photographs of urban scenes, containing buildings, cars, people, etc. After initial presentation of each photograph for 3 seconds each, subjects then perform a two forced choice recognition.

\section{Standardised verbal memory test}

A standardised nonspatial memory test was also included as a control task to assess whether structural differences are associated with good/less good memory performance per se and not selectively with navigational ability. The Adult Memory and Information Processing Battery (AMIPB; Coughlan and Hollows, 1985) includes a test of story recall with norms for a British population. The story is recalled immediately after presentation and then after 30 min of a filled delay. The delayed recall on tests such as this has been found to be sensitive to extent of hippocampal damage (Frisk and Milner, 1990).

\section{Image Acquisition}

For each subject high resolution volumetric MRI was performed on a Siemens 2.0 Tesla Vision scanner (Siemens GmbH, Erlangen, Germany), using an optimised MPRAGE sequence that affords enhanced grey/white matter contrast and segmentation (Deichmann et al., 2000). The acquisition parameters included: TR/ TE/TI 11/4/1000, flip angle 12 , matrix $256 \times 224$, FOV $256 \times$ $224 \mathrm{~mm}$; 176 sagittal slices, $1-\mathrm{mm}$ isotropic voxels.

\section{Image Analysis}

Data were analysed by using whole brain VBM implemented with Statistical Parametric Mapping (SPM99, Wellcome Department of Imaging Neuroscience, London, UK) executed in MATLAB (Mathworks, Shernborn, MA). An optimised method of VBM was used. This method has been described in detail elsewhere (Ashburner and Friston, 2000; Good et al., 2001a,b). Briefly this involves a number of fully automated pre-processing steps including extraction of brain, spatial normalisation into stereotactic space (using the standard SPM grey matter template), segmentation into grey and white matter and CSF compartments, correction for volume changes induced by spatial normalisation, and smoothing with a $10-\mathrm{mm}$ full-width at half-maximum (FWHM) isotropic gaussian kernel. After smoothing, each voxel represents the local average amount of grey (or white) matter in the surrounding region, the size of which is defined by the size of the smoothing kernel. The smoothed data were analysed using SPM99 employing the framework of the General Linear Model. Regionally specific structural differences were assessed statistically in a parametric analysis, namely testing for increases or decreases in grey matter that varied as a function of behavioural performance. Age of subjects was treated as a nuisance covariate in the analysis. Significance levels were set at $P<0.05$, corrected for multiple comparisons (see Results section for description of additional analyses).

\section{RESULTS}

\section{Behavioural Data}

Summary scores for memory performance are shown on Table 1. The inter-correlations between the various measures are shown on Table 2. The two recognition tests, environmental scene recognition and the clinically used CTRMT, correlated with each other. Given that the CTRMT showed limited variance across the 25 subjects who performed it, the environmental scene recognition task was taken to represent recognition in the analysis. Of the experimental navigation tests, only the average angular accuracy and map error scores were correlated. In the human lesion literature the ability to produce sketch maps of an environment and to navigate successfully within it have been reported to dissociate (McCarthy et al., 1996). However, this is likely to be influenced by the type of map test used, whether automated (as in this case) or not. Thus, the precise nature of the relationship between active navigation and map production remains unclear, and correlations between them must be cautiously interpreted and may not be solely spatially based. The fact that no other significant correlations were found between the navigation tasks suggest that they are broadly tapping different aspects of the spatial representation of the VR town, and possibly different regions of the hippocampus. The single subject data for each of the four behavioural measures are shown in Figure 1. The variance in scores is apparent for each measure, confirming the aptness of parametric analyses of expertise in relation to the structural MRI data. 
TABLE 2.

Correlations Between the Behavioural Measures

\begin{tabular}{lccrrr}
\hline & AAcc & EScene rec & Map & CTRMT & DelVrecall \\
\hline AAcc & 1.00 & 0.33 & $-0.51^{* *}$ & 0.24 & 0.17 \\
EScene rec & 0.33 & 1.00 & -0.21 & $0.44^{*}$ & 0.21 \\
Map & $-0.51^{* *}$ & -0.21 & 1.00 & -0.06 & 1.00 \\
CTRMT & 0.24 & $0.44^{*}$ & -0.06 & 0.11 \\
DelVrecall & 0.17 & 0.21 & -0.11 & 0.09 & 1.00 \\
\hline
\end{tabular}

AAcc, average angular accuracy; EScene rec, environmental scene recognition; CTRMT, Camden topographical recognition memory test; DelVrecall, delayed verbal recall.

*Significant at 0.05 level (2-tailed).

**Significant at 0.01 level (2-tailed).

\section{VBM Data: Virtual Town}

\section{Step 1}

Whole brain grey matter volume was examined for changes as a function of level of expertise on any of the three behavioural measures associated with navigation in the virtual town, i.e. average angular accuracy, environmental scene recognition and map error score. Despite the finding of hippocampal grey matter volume differences in the previous taxi driver study using the same technique (Maguire et al., 2000) (Fig. 2A), no significant differences (either increases or decreases) in grey matter volume were found to be related to level of expertise on any of the navigation measures in non-taxi driver subjects.

To minimise the possibility of false-negatives, the data were subjected to further scrutiny as follows.

\section{Step 2}

Because the hippocampus had been identified a priori as a possible candidate for change, the analysis was repeated but with significance levels set at $P<0.005$ uncorrected for multiple comparisons (the intention being to use small volume correction if any hippocampal changes were observed). However, even at this liberal threshold, no significant differences in grey matter volume related to behavioural performance were found.

\section{Step 3}

The above steps treated the performance data parametrically and found no relationship to grey matter volume. The median score was then identified for each behavioural measure and subjects' scores categorised as either above or below the median. For each variable, the two groups of subjects were then compared (in a similar manner to taxi drivers versus control subjects in Maguire et al., 2000) in a categorical fashion, i.e., good versus less good navigators, for any differences in grey mater volume. No significant differences in grey matter volume emerged even at the $P<0.005$ uncorrected level.

\section{Step 4}

Although optimised to use a smoothing kernel of $10 \mathrm{~mm}$ (and hippocampal changes have been reported with a larger kernel of 12 $\mathrm{mm}$ ) (Good et al., 2001b), it might be argued that this is not ideally suited to examination of the hippocampus, and perhaps a smaller smoothing kernel might be more appropriate, such as the 4-mm kernel used by Maguire et al. (2000) and Gadian et al. (2000). Thus, the data were smoothed using a 4-mm kernel, and the analyses (steps 1-3) were repeated. Even at the $P<0.005$ uncorrected level, no significant differences in grey matter volume were found.

\section{Step 5}

Finally, the raw grey matter values were extracted at a voxel in the right posterior hippocampal region. The voxel chosen was that previously identified in the taxi drivers (Maguire et al., 2000) as the peak voxel in this region showing a significant correlation with time taxi driving: $(\mathrm{x}, \mathrm{y}, \mathrm{z}) 22,-33,3$ (Fig. 2B). The raw grey matter data were examined to see if there was any correlation with the performance measures. No significant relationships were found as illustrated in Figure 3, where grey matter volume at the posterior hippocampal voxel is plotted against each of the three VR town measures.

\section{VBM Data: Verbal Memory}

The scores from the delayed recall of the story task were analysed in a similar manner (steps 1-4) to the virtual town measures. In this case also, no significant differences in grey matter volume were found related to the verbal memory measure.

\section{DISCUSSION}

Navigation is a behaviour about which everyone has an opinion, and anecdotes abound about the success or otherwise of attempts to reach a destination. Previous structural brain imaging has shown 


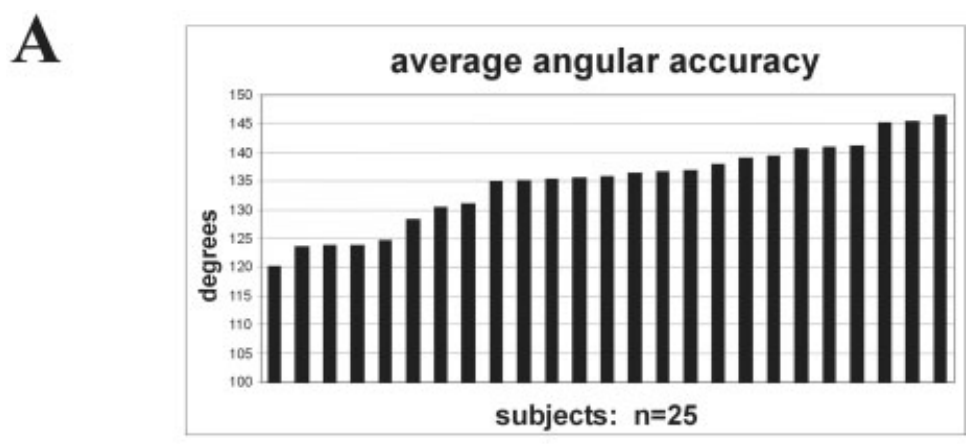

B
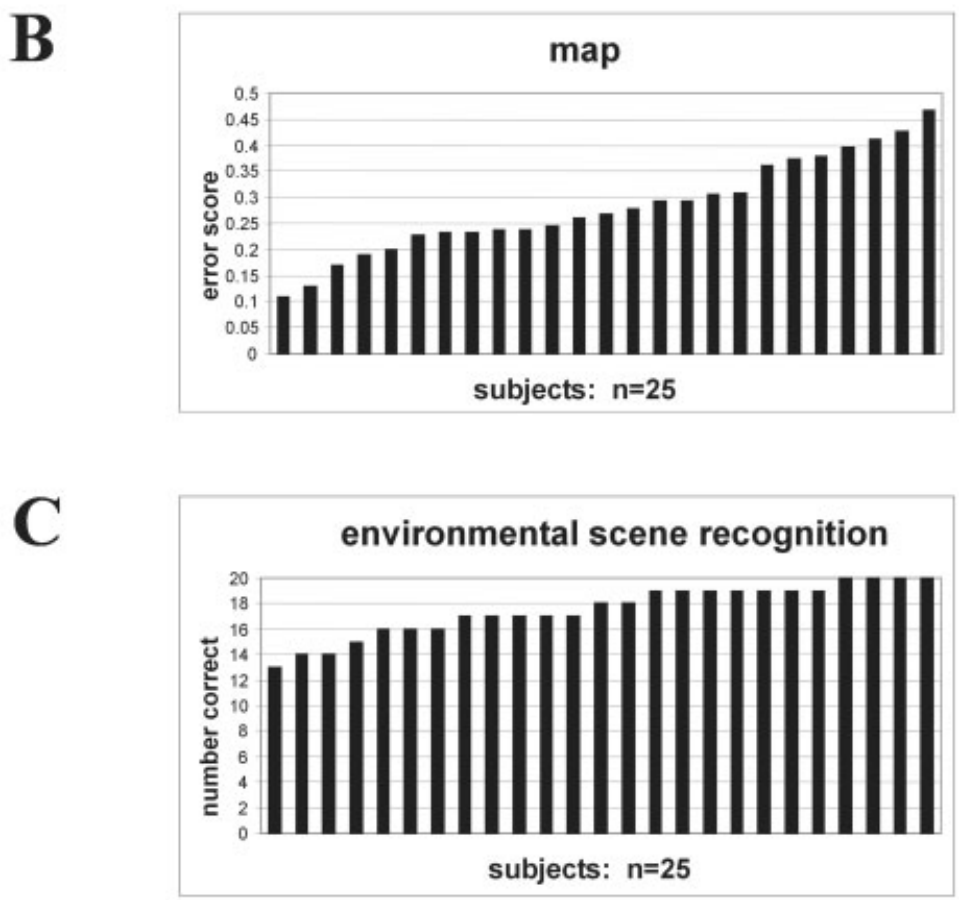

D

FIGURE 1. Performance data for each subject on the behavioural measures. A: Average angular accuracy of navigation in the virtual town (see text). B: Scores on the computerised map production task, where a higher score is indicative of more error (for more details, see Spiers et al., 2001a). C: Scores on the environmental scene recognition test, where the maximum possible is 20. D: scores on the delayed recall of a story (see text), where 56 is the maximum possible score.

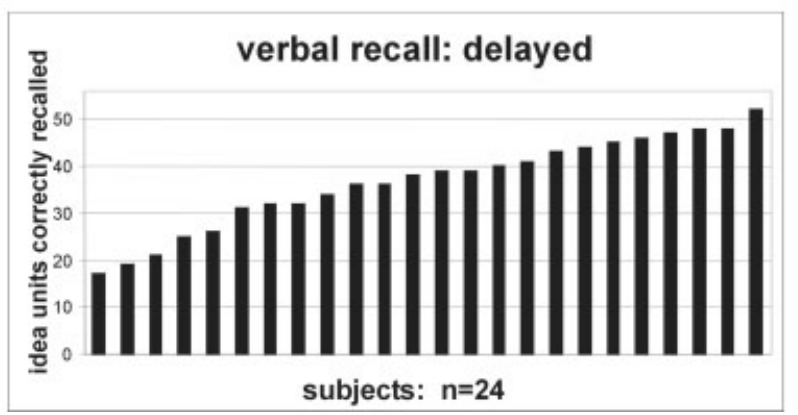

that very expert navigators, London taxi drivers, have greater grey matter volume in the posterior hippocampus compared with control subjects, and for the right posterior hippocampus, grey matter volume varies positively with time taxi driving (Maguire et al., 2000). In the present study, we tested whether navigation expertise in general (i.e., among non-taxi drivers) is reflected in differences in grey matter volume using the same VBM technique that identified the taxi driver changes. Navigation expertise was assessed

using a variety of tasks based in and on a virtual reality town. Despite a wide range of performance on the behavioural measures, no differences in grey matter volume were found to be associated with navigation expertise, even when strenuous efforts were made to minimise the chance of false-negative findings.

Naturally, caution is required in interpreting the absence of positive findings. It may be that the measures used were simply not sensitive enough, although the VBM technique has shown positive 


\section{A}

B
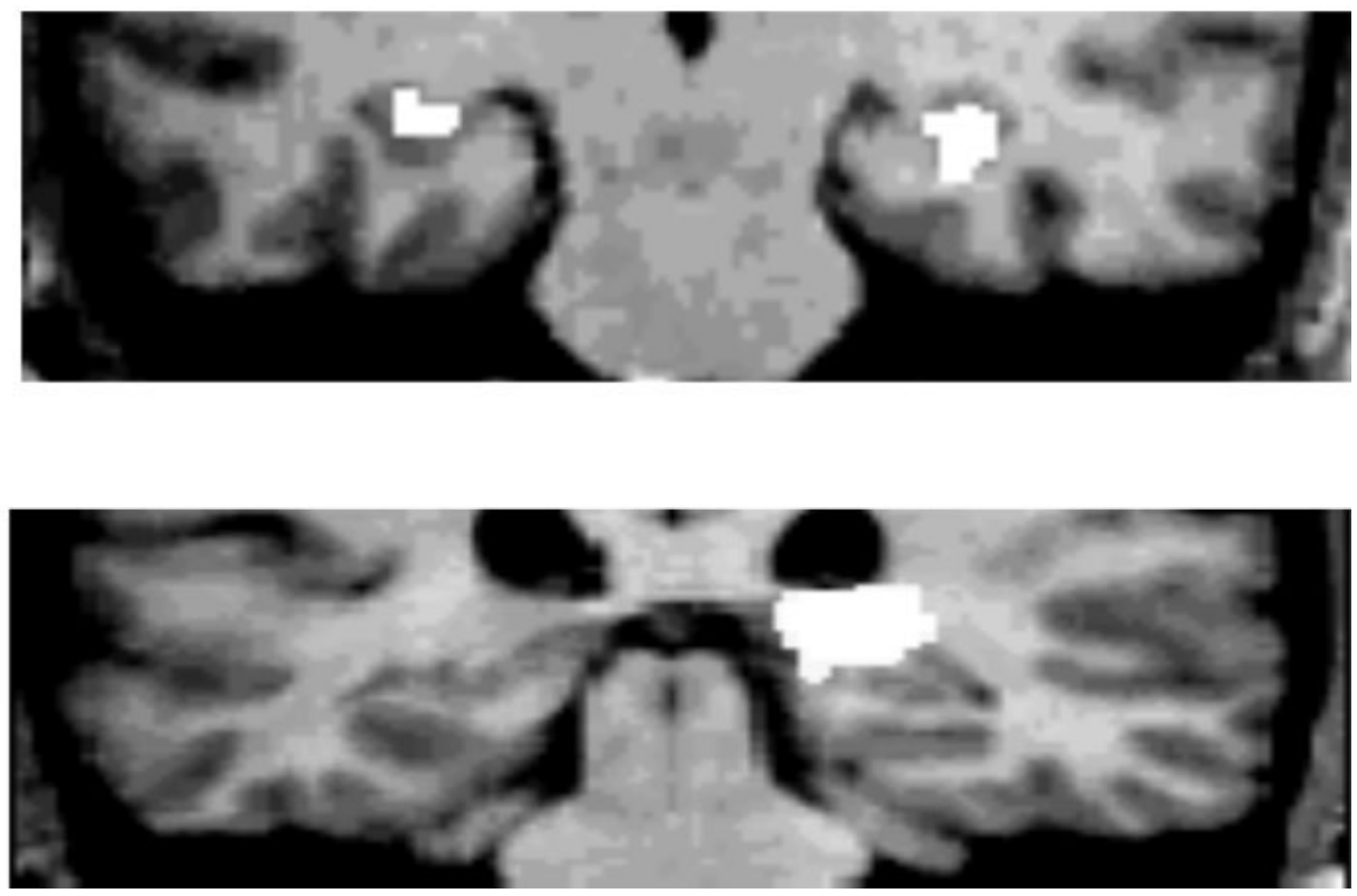

FIGURE 2. Reprise of the voxel-based morphometry (VBM) taxi driver result (originally reported in Maguire et al., 2000). A: Increased grey matter volume in the posterior hippocampi in taxi drivers compared with control subjects. B: Area in the right posterior hippocampal region where the longer the time taxi driving, the greater the grey matter volume. results in relation to the hippocampus in previous studies (e.g., Maguire et al., 2000; Good et al., 2001b; Gadian et al., 2000). Indeed for structural brain analysis, VBM has many advantages over other observer-based region of interest techniques in that it is automated, the whole brain is considered and there are no a priori regions of interest. The MRI scans of the taxi drivers, as well as being analysed using VBM, were also independently analysed using a standard region of interest approach focussing on the hippocampus (Van Paesschen et al., 1997). The findings from the two techniques were completely concordant (Maguire et al., 2000) confirming the appropriateness of using VBM to inform about the hippocampus.

From a previous functional neuroimaging study, successful navigation in the virtual town employed here is known to be associated with right posterior hippocampal activity (Maguire et al., 1998), while average angular accuracy of path may be more sensitive to anterior hippocampus. The cognitive basis of the navigation success associated with posterior activations and how this relates to the more anterior accuracy activity is an open question. Nonetheless, it might be argued that if our measures of navigation expertise are only tapping (anterior hippocampal) accuracy, then the present data do not speak at all to the posterior hippocampal VBM taxi driver result. To mediate this potential problem, however, we included several different town-based tasks. The lack of correlation in general between these tasks suggests that aspects of successful navigation other than path accuracy were being tapped, and possibly hippocampal regions other than the anterior, given that we know that pathology along the length of the hippocampus impairs performance on all of the tasks (Spiers et al., 2001a).

Thus, while acknowledging that the absence of positive findings is not evidence for the absence of an effect, by the same token, one cannot rule out the possibility that there is no effect to be found. We discuss some implications of the latter possibility as supported by the present results.

A lack of association between navigation expertise and structural differences in the hippocampus is reassuring for functional neuroimaging studies. Although activity in the right posterior hippocampus has been associated with successful navigation both in taxi drivers (Maguire et al., 1997) and in control subjects (Maguire et al., 1998; Ghaem et al., 1997; Grön et al., 2000), it now appears this is not simply due to more expert navigators having more hippocampal tissue to activate. It would seem that for ability at least, size is not the issue for the hippocampus, but perhaps how it is used. If innate navigation expertise is not the cause of structural brain differences, what is the basis of the changes recorded in the taxi drivers (Maguire et al., 2000)? It cannot be merely that they are successful and accurate navigators, as so were many of the present non-taxi driver subjects. Rather, the present findings support the interpretation that has been previously elaborated to account for increased hippocampal volume in the taxi drivers (Maguire et al., 2000). What differs among the taxi drivers is not accuracy, but rather the nature of their spatial representation of the city. They themselves describe that over time it becomes more integrated, more survey-like, permitting increasing understanding of how 
A

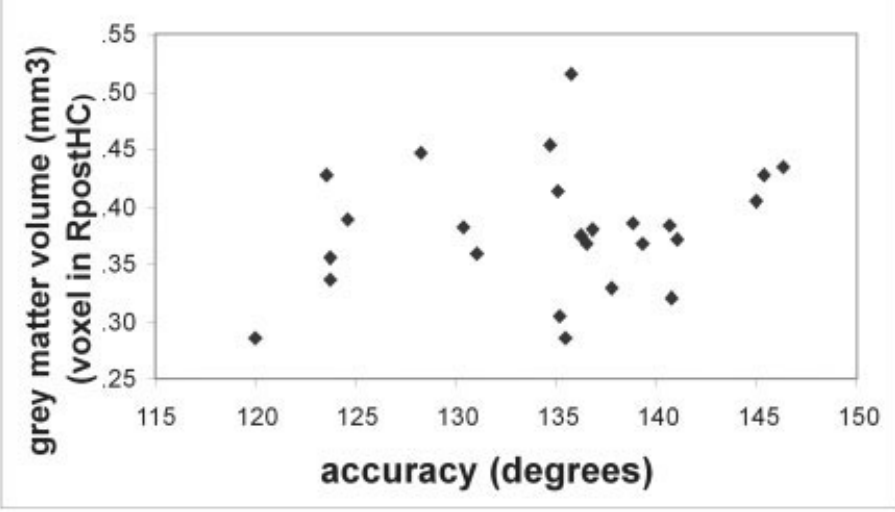

B

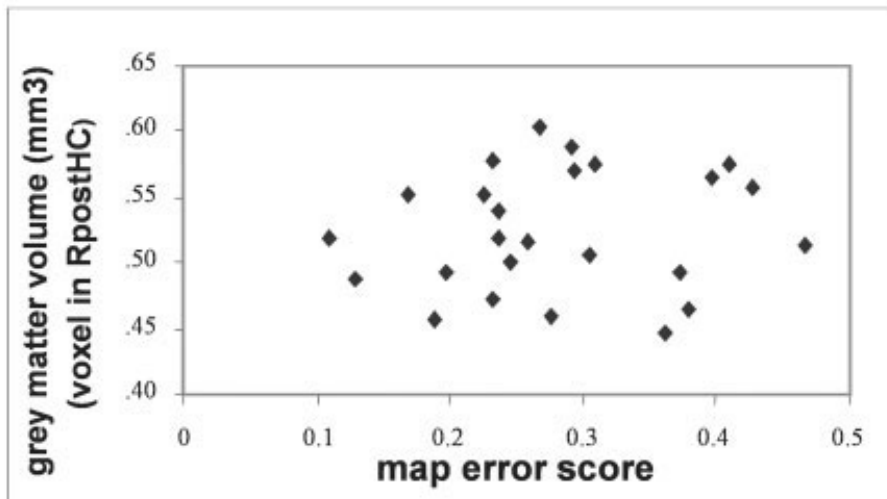

FIGURE 3. Correlation between navigation expertise and grey matter volume. Plots of the three virtual reality town-based measures against grey matter values extracted from a voxel in the right posterior hippocampal region, chosen based on the previously observed finding in the taxi drivers of the correlation of grey matter volume with time taxi driving (see Maguire et al., 2000, and Fig. 2b). There were no significant correlations between any of the behavioural measures and the grey matter values for $(A)$ accuracy $(r=0.2)$, (B) map score $(r=0.1)$, or (C) environmental scene recognition $(r=0.03)$.

routes and places relate to each other (E.A. Maguire, unpublished observations).

In functional terms, it may be that the previously-noted activity in the right posterior hippocampus in both taxi drivers and control subjects reflected the use of the allocentric survey-like representation of the environment stored in this brain region. There may be further support for this from a study reported by Mellet et al. (2000). Using functional neuroimaging, they examined what brain

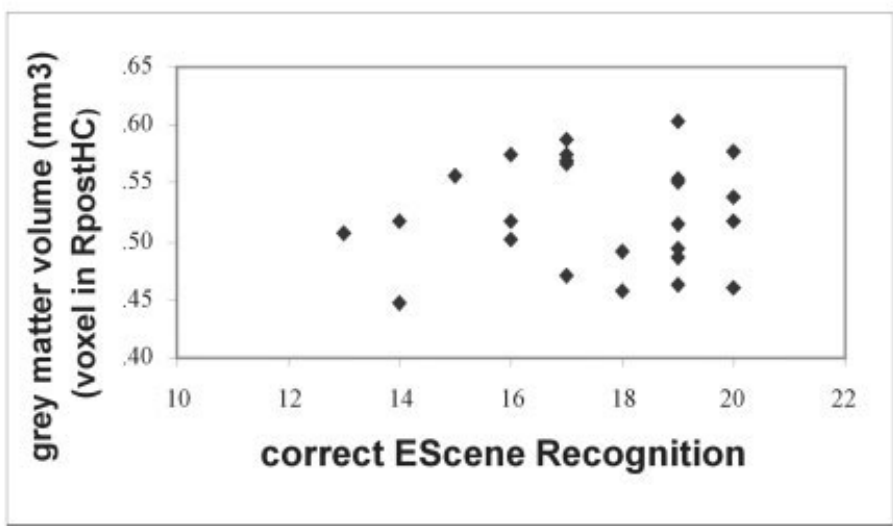

regions were active in common when an environment had been either learned from direct navigation or by means of a survey-like map. These investigators found that the right posterior hippocampus was common to both types of representation and suggested that the posterior hippocampus may represent survey (allocentric)like information initially acquired in a route perspective. In structural terms, consolidation and use of spatial information on the scale of the taxi drivers is very difficult to test in the general popu- 
lation as we are rarely in possession of such a detailed spatial representation, and our hippocampi are probably more than able to cope with our typical needs without recourse to structural change. However, there may be a threshold (either in terms of detail or duration of use) beyond which storage and elaboration of a largescale spatial representation induces hippocampal plasticity.

The notion of environmental stimulation being associated with greater grey matter volume seems to imply some form of cell proliferation. It is now widely acknowledged that neurogenesis occurs in the adult brain in two brain regions, the olfactory system but also the hippocampus (for review, see Gross, 2000). In the hippocampus, morphological, biochemical and ultrastructural evidence indicate that these newly-generated neurons can become incorporated into the preexisting neural circuitry of the dentate gyrus (Hastings et al., 2001) displaying membrane and synaptic properties similar to those found in mature cells (van Praag et al., 2002). There is now further evidence that these cells have some functional significance. Physical activity and enriched living environments have been found to result in increased neurogenesis in the rodent hippocampus (e.g., van Praag et al., 1999; Kempermann et al., 1997; Gould et al., 1999; see also van Praag et al., 2000, for review). Of particular relevance to navigation, it has also been recently demonstrated that reducing the number of newly generated neurons in the adult rat hippocampus, impairs hippocampaldependent forms of associative memory formation (Shors et al., 2001).

Much of the aforementioned evidence pertains to the rodent hippocampus, although neurogenesis has also been documented in the primate hippocampus (e.g., Kornak and Rakic, 1999) and has also been observed in post-mortem human hippocampal tissue (Eriksson et al., 1998), although its functional significance is as yet undetermined. While much remains to be understood about neurogenesis (see Gould and Gross, 2002; Kempermann, 2002; Macklis, 2001), the extant evidence is completely concordant with the VBM findings both in the taxi drivers and those reported here.

In summary, the present findings speak further to issues such as innate versus acquired differences, expertise, and consolidation raised by the previous structural study (Maguire et al., 2000). Notwithstanding the caveats noted above, this study permits continued support for the view that there is a capacity for local plastic change in the human brain in response to environmental stimulation, and specifically in the right posterior hippocampus in relation to the representation of large-scale space. It also highlights the advantage of using functional and structural brain imaging and of neuropsychological studies in the context of realistic but controlled large-scale (possibly virtual) environments to gain complimentary insights into how the human hippocampus supports navigation.

\section{Acknowledgments}

This work was supported by the Wellcome Trust (to E.A.M., C.D.G, R.S.J.F.); and by the Medical Research Council, UK (to H.J.S., T.H., N.B.). The authors thank Geraint Rees for helpful comments.

\section{REFERENCES}

Ashburner J, Friston KJ. 2000. Voxel-based morphometry-the methods. NeuroImage 11:805-821.

Burgess N, King, JA. 2001. Navigation in virtual space: psychological and neural aspects. In: Smelser NJ, Baltes PB, editors. International encyclopaedia of social and behavioral sciences. Amsterdam: Elsevier. Vol. 15, p 10417-10422.

Burgess N, Maguire EA, Spiers H, O'Keefe J. 2001. A temporoparietal and prefrontal network for retrieving the spatial context of lifelike events. NeuroImage 14:439-453.

Coughlan A, Hollows SE. 1985. Adult memory and information processing battery. Psychology Department, St James Hospital, Leeds, UK.

Deichmann R, Good CD, Josephs O, Ashburner J, Turner R. 2000. Optimization of 3D MP-RAGE sequences for structural brain imaging. NeuroImage 12:112-127.

Eriksson PS, Perfilieva E, Bjork-Eriksson T, Alborn, A-M, Nordborg C, Peterson DA, Gage FH. 1998. Neurogenesis in the adult human hippocampus. Nature Med 4:1313-1317.

Frisk V, Milner B. 1990. The role of the left hippocampal region in the acquisition and retention of story content. Neuropsychologia 28:349359.

Gadian DG, Aicardi J, Watkins KE, Porter DA, Mishkin M, VarghaKhadem F. 2000. Developmental amnesia associated with early hypoxic-ischaemic injury. Brain 123:499-507.

Ghaem O, Mellet E, Crivello F, Tzourio N, Mazoyer B, Bethoz A, Denis M. 1997. Mental navigation along memorized routes activates the hippocampus, precuneus, and insula. NeuroReport 8:739-744.

Good CD, Johnsrude IS, Ashburner J, Henson RNA, Friston KJ, Frackowiak RSJ. 2001a. A voxel-based morphometric study of ageing in 465 normal adult human brains. NeuroImage 14:21-36.

Good CD, Johnsrude IS, Ashburner J, Henson RNA, Friston KJ, Frackowiak RSJ. 2001b. Cerebral asymmetry and the effects of sex and handedness on brain structure: a voxel based morphometric analysis of 465 normal adult human brains. NeuroImage 14:685-700.

Gould E, Gross CG. 2002. Neurogenesis in adult mammals: some progress and problems. J Neurosci 22:619-623.

Gould E, Beylin A, Tanapat P, Reeves A, Shors T. 1999. Learning enhances adult neurogenesis in the hippocampal formation. Nature Neurosci 2:260-265.

Grön G, Wunderlich AP, Spitzer M, Tomczak R, Riepe MW. 2000. Brain activation during human navigation: gender-different neural networks. Nature Neurosci 3:404-408.

Gross CG. 2000. Neurogenesis in the adult brain: death of a dogma. Nature Rev 1:67-73.

Hastings NB, Tanapat P, Gould E. 2001. Neurogenesis in the adult mammalian brain. Clin Neurosci Res 1:175-182.

Hermer L, Spelke ES. 1994. A geometric process for spatial reorientation in young children. Nature 370:57-59.

Kempermann G. 2002. Why new neurons? Possible functions for adult hippocampal neurogenesis. J Neurosci 22:635-638.

Kempermann G, Kuhn HG, Gage FH. 1997. More hippocampal neurons in adult mice living in an enriched environment. Nature 386:493495.

Kornack DR, Rakic P. 1999. Continuation of neurogenesis in the hippocampus of the adult macaque monkey. Proc Natl Acad Sci U S A 96:5768-5773.

Lee DW, Miyasato LE, Clayton NS. 1998. Neurobiological bases of spatial learning in the natural environment: neurogenesis and growth in the avian and mammalian hippocampus. NeuroReport 9:R15-R27.

Macklis JD. 2001. New memories from new neurons. Nature 410:314317.

Maguire EA, Frackowiak RSJ, Frith CD. 1997. Recalling routes around London: activation of the right hippocampus in taxi drivers. J Neurosci 17:7103-7110. 
Maguire EA, Burgess N, Donnett JG, Frackowiak RSJ, Frith CD, O'Keefe J. 1998. Knowing where and getting there: a human navigation network. Science 280:921-924.

Maguire EA, Burgess N, O'Keefe J. 1999. Human spatial navigation: cognitive maps, sexual dimorphism, and neural substrates. Curr Opin Neurobiol 9:171-177.

Maguire EA, Gadian DG, Johnsrude IS, Good CD, Ashburner J, Frackowiak RSJ, Frith CD. 2000. Navigation-related structural change in the hippocampi of taxi drivers. Proc Natl Acad Sci U S A 97:43984403.

McCarthy RA, Evans JJ, Hodges JR. 1996. Topographic amnesia: spatial memory disorder, perceptual dysfunction, or category specific semantic memory impairment? J Neurol Neurosurg Psychol 90:318-325.

Mellet E, Bricogne S, Tzourio-Mazoyer, Ghaem O, Petit L, Zago L, Etard O, Berthoz A, Mazoyer B, Denis M. 2000. Neural correlates of topographic mental exploration: the impact of route versus survey perspective learning. NeuroImage 12:588-600.

Nelson HE, Wilson JR. 1991. National Adult Reading Test (NART). Test manual. 2nd ed. Windsor: Berkshire.

O'Keefe J, Nadel L. 1978. The hippocampus as a cognitive map. Oxford: Oxford University Press.

O’Neill MJ. 1992. Effects of familiarity and plan complexity on wayfinding in simulated buildings. J Environ Psychol 12:319-327.

Prestopnik JL, Roskos-Ewoldsen B. 2000. The relations among wayfinding strategy use, sense of direction, sex, familiarity, and wayfinding ability. J Environ Pychol 20:177-191.

Regan EC, Price KR. 1994. The frequency of occurrence and severity of side-effects of immersion virtual reality. Aviat Space Environ Med 65:527-530.

Sandstrom NJ, Kaufman J, Huettel SA. 1998. Males and females use different distal cues in a virtual environment navigation task. Brain Res Cogn 6:351-360.
Shors T, Miesegaes G, Beylin A, Zhao M, Rydel T, Gould E. 2001. Neurogenesis in the adult is involved in the formation of trace memories. Nature 410:372-376.

Siegal AW, White SH. 1975. The development of spatial representation in of large-scale environments. In: Reese HW, editor. Advances in child development and behavior. New York: Academic Press. p 1055.

Spiers HJ, Burgess N, Maguire EA, Baxendale SA, Hartley T, Thompson P, O'Keefe J. 2001a. Unilateral temporal lobectomy patients show lateralised topographical and episodic memory deficits in a virtual town. Brain 124:2476-2489.

Spiers HJ, Burgess N, Hartley T, Vargha-Khadem F, O’Keefe J. 2001b. Bilateral hippocampal pathology impairs topographical and episodic but not recognition memory. Hippocampus 11:715-725.

Terrazas A, McNaughton BL. 2000. Brain growth and the cognitive map. Proc Natl Acad Sci U S A 97:4414-4416.

Tlauka M, Wilson PN. 1994. The effect of landmarks on route-learning in a computer-simulated environment. J Environ Pychol 14:305-313.

Van Paesschen W, Connolly A, King MD, Jackson GD, Duncan JS. 1997. The spectrum of hippocampal sclerosis: a quantitative magnetic resonance imaging study. Ann Neurol 41:41-51.

van Praag H, Christie BR, Sejnowski TJ, Gage FH. 1999. Running enhances neurogenesis, learning, and long-term potentiation in mice. Proc Natl Acad Sci U S A 96:13427-13431.

van Praag H, Kempermann G, Gage FH. 2000. Neural consequences of environmental enrichment. Nature Rev 1:191-198.

van Praag H, Schnider AF, Christie BR, Toni N, Palmer TD, Gage FH. 2002. Functional neurogenesis in the adult hippocampus. Nature 415: 1030-1034.

Vargha-Khadem F, Gadian DG, Watkins KE, Connelly A, Van Paesschen W, Mishkin M. 1997. Differential effects of early hippocampal pathology on episodic and semantic memory. Science 277:376-380.

Warrington EK. 1996. The Camden Memory Tests. Psychology Press. 